\title{
Prevention, early detection, and management of complications after 328 consecutive extrapleural pneumonectomies
}

\author{
David J. Sugarbaker, $M D^{a}$ \\ Michael T. Jaklitsch, MD \\ Raphael Bueno, MD ${ }^{\mathrm{a}}$ \\ William Richards, $\mathrm{PhD}^{\mathrm{a}}$ \\ Jeanne Lukanich, $\mathrm{MD}^{\mathrm{a}}$ \\ Steven J. Mentzer, MD ${ }^{\mathrm{a}}$ \\ Yolonda Colson, MD, $\mathrm{PhD}^{\mathrm{a}}$ \\ Phillip Linden, $\mathrm{MD}^{\mathrm{a}}$ \\ Michael Chang, MD \\ Leah Capalbo, $\mathrm{BA}^{\mathrm{a}}$ \\ Elizabeth Oldread, MPH ${ }^{\mathrm{a}}$ \\ Siyamek Neragi-Miandoab, MD \\ Scott J. Swanson, MD ${ }^{\mathrm{b}}$ \\ Lambros S. Zellos, MD, MPH ${ }^{\mathrm{a}}$
}

From the Division of Thoracic Surgery, Brigham and Women's Hospital, Boston, Mass, and the Division of Thoracic Surgery, ${ }^{\mathrm{b}}$ Mt Sinai Hospital and Medical Center, New York, NY.

Read at the Eighty-third Annual Meeting of The American Association for Thoracic Surgery, Boston, Mass, May 4-7, 2003.

Received for publication June 17, 2003; revisions received Feb 2, 2004; accepted for publication Feb 10, 2004.

Address for reprints: David J. Sugarbaker, MD, Division of Thoracic Surgery, Brigham and Women's Hospital, 75 Francis St, Boston MA 02115 (E-mail: dsugarbaker@partners.org).

J Thorac Cardiovasc Surg 2004;128:138-46 $0022-5223 / \$ 30.00$

Copyright $\odot 2004$ by The American Association for Thoracic Surgery

doi:10.1016/j.jtcvs.2004.02.021

\begin{abstract}
Objective: Extrapleural pneumonectomy for therapy of mesothelioma has been associated with significant perioperative mortality and morbidity. Postoperative complications of this procedure require a unique management approach. We developed treatment algorithms for most of the common complications of extrapleural pneumonectomy resulting in reduced mortality and hospital stay. Complications after extrapleural pneumonectomy were further analyzed to elucidate means of prevention, early detection, and treatment.
\end{abstract}

Methods: A total of 496 patients undergoing extrapleural pneumonectomy were reviewed for mortality rates, with a subset of 328 consecutive patients between 1980 and 2000 who were examined for detailed morbidity data by using a prospective clinical database.

Results: Median age was 58 years (range, 28-77 years), with a 10-day (range, 4-101 days) median length of stay. One hundred ninety-eight (60.4\%) of 328 patients experienced minor and major complications, and 11 of 328 patients died, for an overall mortality rate of $3.4 \%$. Complications included the following: atrial fibrillation (145 [44.2\%]), prolonged intubation (26 [7.9\%]), vocal cord paralysis (22 [6.7\%]), deep vein thrombosis (21 [6.4\%]), technical complications (patch dehiscence, hemorrhage, or both; $20[6.1 \%])$, tamponade (12 [3.6\%]), acute respiratory distress syndrome (12 [3.6\%]), cardiac arrest (10 [3\%]), constrictive physiology (9 $[2.7 \%])$, aspiration $(9[2.7 \%])$, renal failure $(9[2.7 \%])$, empyema $(8[2.4 \%])$, tracheostomy (6 $[1.8 \%])$, myocardial infarction $(5[1.5 \%])$, pulmonary embolus (5 $[1.5 \%])$, and bronchopleural fistula $(2[0.6 \%])$. Clinical data demonstrated the following: (1) prophylaxis for atrial fibrillation is recommended; (2) early ambulation, aspiration precautions, endoscopic assessment of the vocal cords, and avoidance of fluid overload are crucial; (3) perioperative diagnosis and aggressive management of deep vein thrombosis are important; (4) immediate reoperation and open cardiac massage are essential for relief of cardiac herniation and tamponade from cardiac patch dysfunction; (5) diaphragmatic patch dehiscence, hemorrhage, or both require immediate reoperation; (6) early signs of infection might indicate bronchopleural fistula or empyema and should be treated with thoracoscopic or open 
drainage and staged removal of patch material; and (7) excessive perioperative mediastinal shift is treated with a catheter placed intraoperatively.

Conclusion: Complications after extrapleural pneumonectomy require a unique approach to management, and mortality can be minimized by early detection and aggressive treatment.

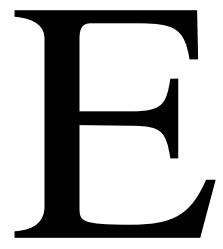

xtrapleural pneumonectomy (EPP) was introduced in the 1940s for the treatment of extensive infections of the lung and pleural space, such as tuberculous empyema. Over the past 20 years, we have applied EPP to treat locally advanced malignant pleural mesothelioma (MPM).

Various centers have reported their techniques and results with EPP, with particular reference to the management of MPM. In 1976, Butchart and colleagues ${ }^{1}$ reported their experience with this operation in patients with mesotheliomas and proposed a staging system on the basis of these results. Although this study remains one of the most quoted in the literature, an extremely poor operative mortality of $31 \%$ was reported. Several other series have since reported operative results of EPP, with operative mortalities ranging between $8 \%{ }^{2}$ and $15 \% .^{3}$ In 1999 , we reported a series of 183 consecutive patients with MPM who had undergone EPP, with a mortality rate of $3.8 \% .{ }^{4}$ Since that time, we have expanded our use of this operation to treat locally advanced lung cancer and thymoma. Additional refinements in technique and management have been implemented since 1999, resulting in still further reductions in operative mortality.

EPP is a technically challenging operation accompanied by a high rate of perioperative morbidity. Safe outcomes require a multidisciplinary team approach and skills to appreciate the significance of early signs of complications. Our knowledge of the anticipated complications and interventions to prevent and minimize the effect of these complications has been developed largely through personal experience. As a result, we currently recognize more perioperative morbidity, but aggressive intervention has led to reduced perioperative mortality. The purpose of this article is to provide a detailed description of the potential postoperative complications of EPP and our institutional approach to postoperative management.

\section{Methods}

In a retrospective review of medical records at our institution, we identified patients who underwent EPP between 1980 and 1989 to supplement a prospective database that was created in 1990. The retrospective data were collected from medical records from the 1980s when the database was first created in 1990. Prospective data have been collected since 1990 through patient question-
TABLE 1. Patient selection criteria

Patient selection criteria

Karnofsky performance

Renal function

Liver function

Pulmonary function

$>70$

Creatinine $<2$

AST $<80 \mathrm{IU} / \mathrm{L}$, total bilirubin $<1.9 \mathrm{mg} / \mathrm{dL}$, PT $<15 \mathrm{~s}$

Postoperative $\mathrm{FEV}_{1}>0.8 \mathrm{~L}$ as per PFTs and quantitative ventilation-perfusion scans

Cardiac function

Grossly normal cardiac function as per ECG and echocardiography (EF preferably $>45 \%$ )

Extent of disease

Limited to ipsilateral hemithorax with no transdiaphragmatic, transpericardial, or extensive chest wall involvement

$\overline{A S T}$, Aspartate aminotransferase; $P T$, prothrombin time; $F E V_{1}$, forced expiratory volume in 1 second; PFTs, pulmonary function tests; ECG, electrocardiography; $E F$, ejection fraction.

naires, as well as visits to the operating rooms, postoperative wards, and outpatient clinics by our surgical data manager. Institutional review board approval for the collection of outcome data in compliance with HIPPA regulations was obtained as early as 1998.

This medical record review revealed that 496 patients had EPP at the Brigham and Women's Hospital between 1980 and March 2003. Selection criteria for EPP have not changed since our previous publication (Table 1$){ }^{4}$

\section{Results}

From 1980 through 2003, 496 consecutive patients underwent EPP at the Brigham and Women's Hospital in Boston. Of this total, 444 patients underwent EPP for mesothelioma, 28 for stage IIIB adenocarcinoma, 4 for thymoma, and 20 for other diseases. There were 20 deaths within the first 30 postoperative days or within the same hospitalization, for an overall operative mortality of $4.0 \%$. Pulmonary embolus was the most common cause of mortality. The 3 patients who died of unknown causes were from out of state. Although we know they died during the postoperative period, we are unable to ascertain the exact cause of death (Table 2). 
TABLE 2. Cause of death

\begin{tabular}{lc}
\hline Cause & $\begin{array}{c}\text { No. of patients } \\
\text { ( } \mathbf{n}=\mathbf{2 0 / 4 9 6 )}\end{array}$ \\
\hline Pulmonary embolus & 6 \\
ARDS & 4 \\
Myocardial infarction & 3 \\
Unknown & 3 \\
Cardiac herniation & 1 \\
Renal failure & 1 \\
Cardiac arrhythmia & 1 \\
Heparin-induced & 1 \\
thrombocytopenia & \\
\hline
\end{tabular}

ARDS, Acute respiratory distress syndrome.

Detailed morbidity data were accumulated for the first 328 patients with mesotheliomas undergoing operation up to the year 2000 (Figure 1). Age ranged from 28 to 77 years, with a median age of 58 years. The overall minor and major morbidity rate was $60.4 \%$. Median length of stay was 10 days, with a range of 4 to 101 days (Table 3).

\section{Cardiac Complications}

Atrial fibrillation (AF) was the most common cardiac and overall complication in this cohort, with 145 (44.2\%) of 328 patients having episodes of AF. Although we have implemented several different prophylactic strategies to address $\mathrm{AF}$, none has successfully reduced the frequency of this source of morbidity, and we must await randomized studies to elucidate an effective prevention regimen in this patient group.

Other more serious but less common complications include constrictive physiology caused by inflammatory epicarditis (9/328 [2.7\%] patients), with 8 patients requiring reoperation; cardiac tamponade (12/328 [3.6\%] patients), myocardial infarction (5/328 [1.5\%] patients); and cardiac arrest (10/328 [3\%] patients).

All cases of constrictive physiology occurred after left EPP when we did not routinely reconstruct the left pericardium. All patients were symptomatic and had evidence of constrictive physiology either determined by means of cardiac catheterization or by means of echocardiography. To combat this problem, we began to place a patch over the pericardial defect, even after left EPP, and since using this practice, we have not encountered a single additional case. The 8 patients requiring reoperation had inflammatory epicarditis as the source of their constrictive physiology. ${ }^{5}$

Cardiac tamponade physiology can be either the result of retained pericardial effusion or constrictive patches. Although tamponade arising from pericardial effusion is high on the list of differential diagnoses, the echocardiographer should also document the diastolic function of the right ventricle. A tight or inadequately fenestrated pericardial patch can also produce tamponade physiology because of
TABLE 3. Post-EPP morbidities $(\mathrm{n}=\mathbf{3 2 8})$

\begin{tabular}{|c|c|}
\hline Age & Median, 58 y; range, 28-77 y \\
\hline Length of stay & Median, $10 \mathrm{~d}$; range, 4-101 d \\
\hline Complications* & \\
\hline $\begin{array}{c}\text { Minor and major } \\
\text { morbidity rate }\end{array}$ & $60.4 \%(198 / 328)$ \\
\hline Atrial fibrillation & $44.2 \%(145 / 328)$ \\
\hline Myocardial infarction & $1.5 \%(5 / 328)$ \\
\hline Constrictive physiology & $2.7 \%(9 / 328)$ \\
\hline $\begin{array}{l}\text { Reoperation for } \\
\text { constrictive } \\
\text { physiology }\end{array}$ & $2.4 \%(8 / 328)$ \\
\hline Tamponade & $3.6 \%(12 / 328)$ \\
\hline Cardiac arrest & $3 \%(10 / 338)$ \\
\hline Prolonged intubation & $7.9 \%(26 / 328)$ \\
\hline Aspiration & $2.7 \%(9 / 328)$ \\
\hline ARDS & $3.6 \%(12 / 328)$ \\
\hline Tracheostomy & $1.8 \%(6 / 328)$ \\
\hline Vocal cord paralysis & $6.7 \%(22 / 328)$ \\
\hline Renal failure & $2.7 \%(9 / 328)$ \\
\hline Deep vein thrombosis & $6.4 \%(21 / 328)$ \\
\hline Pulmonary embolus & $1.5 \%(5 / 328)$ \\
\hline $\begin{array}{l}\text { CVA (33 days } \\
\text { postoperative) }\end{array}$ & $0.3 \%(1 / 328)$ \\
\hline Empyema & $2.4 \%(8 / 328)$ \\
\hline Bronchopleural fistula & $0.6 \%(2 / 328)$ \\
\hline $\begin{array}{l}\text { Technical complications } \\
\text { (patch failure and } \\
\text { bleeding) }\end{array}$ & $6.1 \%(20 / 328)$ \\
\hline Ischemic colitis grade II & $0.3 \%(1 / 328)$ \\
\hline lleus & $0.9 \%(3 / 328)$ \\
\hline $\begin{array}{l}\text { Colectomy for } \\
\text { Clostridium difficile }\end{array}$ & $0.3 \%(1 / 328)$ \\
\hline
\end{tabular}

ARDS, Acute respiratory distress syndrome; CVA, cardiovascular accident. *Percentage of patients ( $\mathrm{n} /$ total).

the inability of the ventricle to fill during diastole. Likewise, a diaphragmatic patch that impinges on the inferior vena cava, especially when the heart displaces into the right chest after a right EPP, will produce the same lack of ventricular filling. An important clue to this condition arises in the operating room when the patient is turned from the lateral decubitus to the supine position. A tight patch will result in persistent hypotension with an increase of the filling pressures (Figure 2). The treatment of choice is to re-explore the patient and reconstruct the pericardium immediately with a looser patch. Transesophageal echocardiography can assist in making the diagnosis before leaving the operating room. The best treatment is prevention, and a tight patch can be avoided by beginning the reconstruction in the posterior pericardium, which tends to be deep, and is thus the most difficult part of the reconstruction. The posterior pericardium with the sutured patch retracts posteriorly, therefore permitting better sizing of the patch when it is sutured to the anterior pericardium. For additional laxity, the inferior border of the pericardial patch can be sewn to the diaphragmatic patch about $1 \mathrm{~cm}$ lateral to the suture line, uniting the 


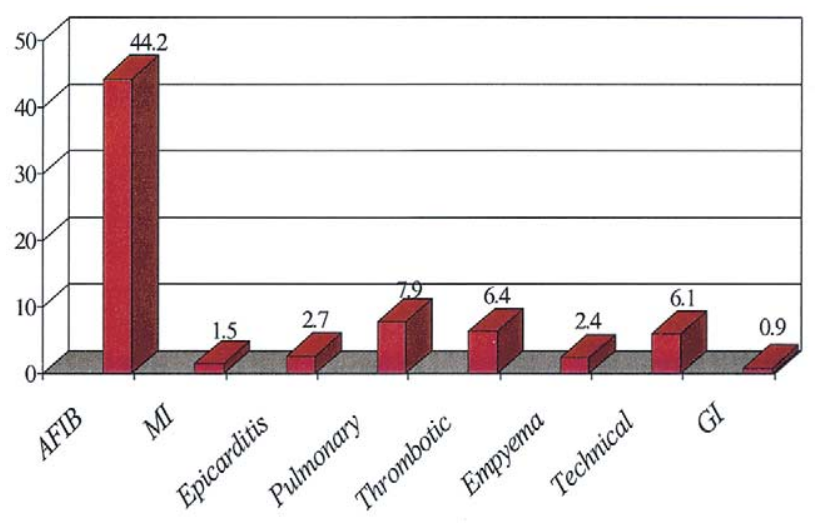

Figure 1. Morbidity distribution (\%; $n=328$ ). AFIB, Atrial fibrilIation; MI, myocardial infarction; GI, gastrointestinal.

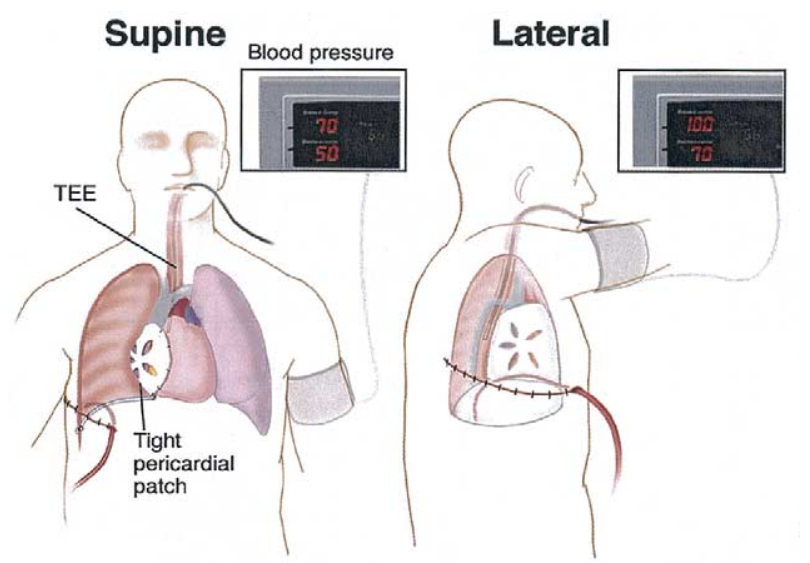

Figure 2. A tight pericardial patch can present with hypotension on turning the patient from the lateral to the supine position. The surgeon must be present in the operating room when the patient is being turned because major hemodynamic instability can occur. TEE, Transesophageal echocardiography.

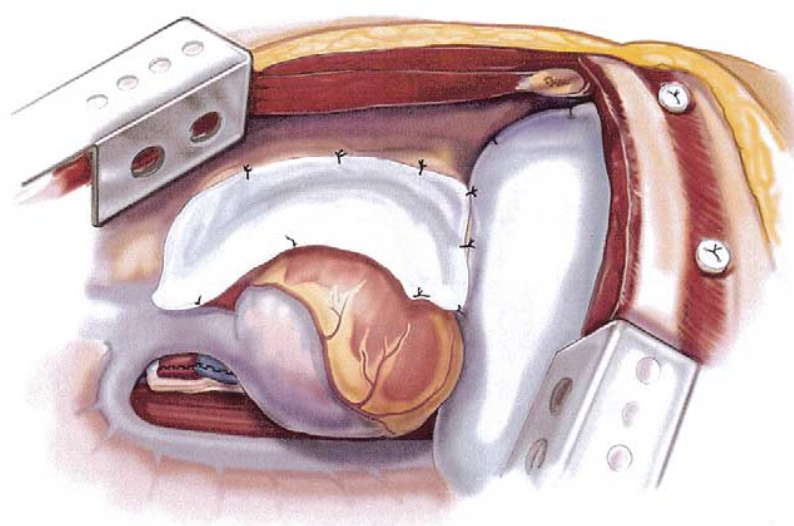

Figure 3. A pericardial patch that has not been anchored to the pericardial edge can result in cardiac herniation.

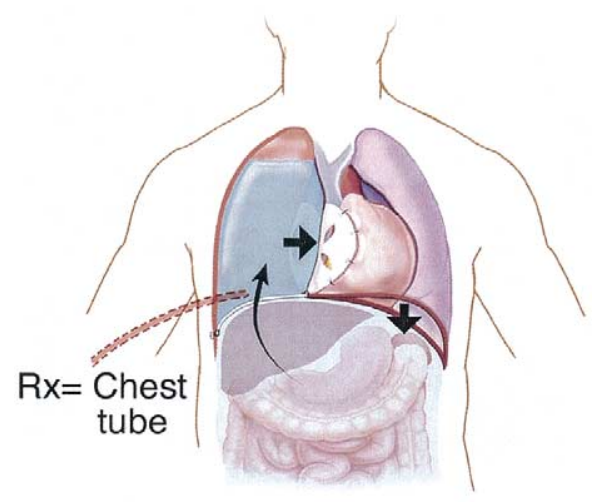

Figure 4. Excessive mediastinal shift away from the pneumonectomy space into the contralateral hemithorax can result in respiratory compromise. A red rubber catheter is left in the pneumonectomy space to evacuate fluid as needed.

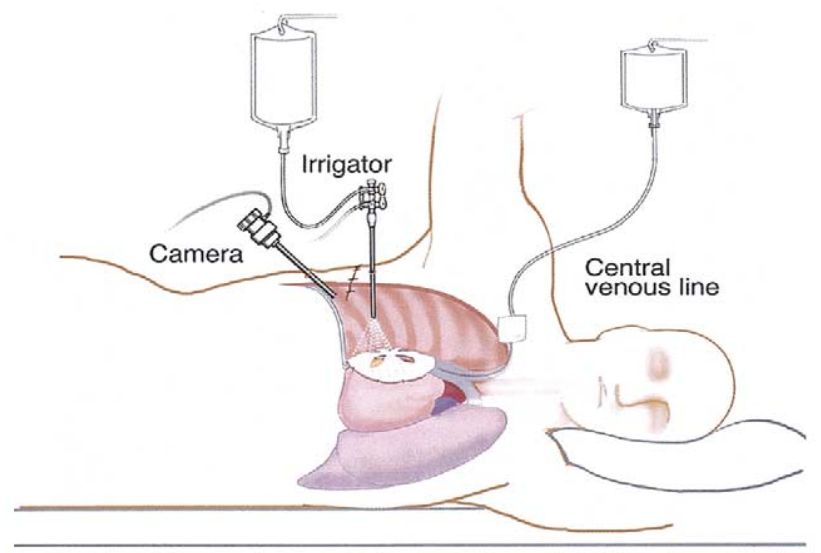

Figure 5. Thoracoscopic drainage with full debridement of all fibrin and pulsed irrigation of the pleural cavity without patch removal is being used for treatment of early empyema. This is followed by antibiotic intracavitary irrigation for $\mathbf{5}$ days.

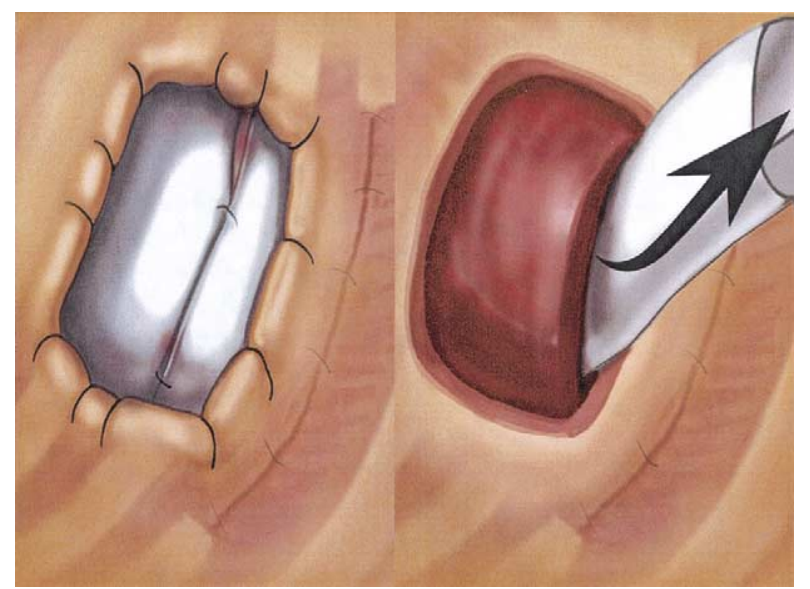

Figure 6. If a Clagett window is deemed necessary, then staged removal of the prosthetic PTFE patches can prevent overshift of the mediastinum in the early postoperative period. 


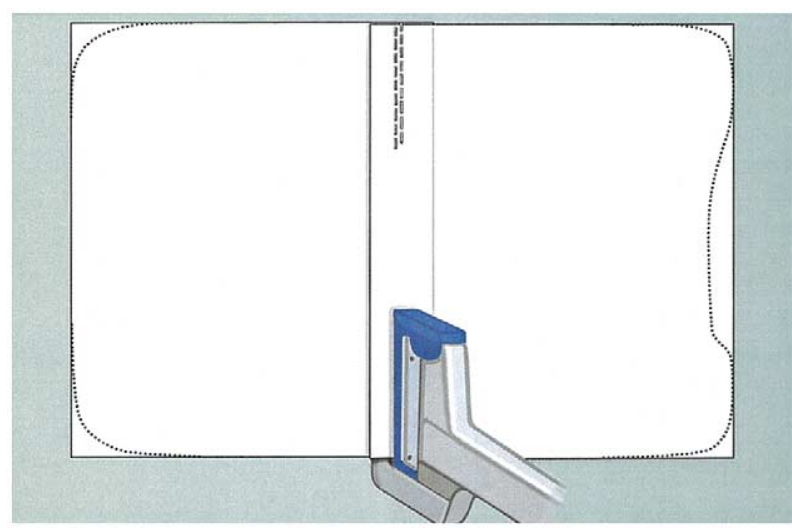

Figure 7. Two patches are used and stapled with a TA stapler, creating a dynamic patch at the center with less tension at the sutures that can better accommodate the abdominal contents. The patch is then tailored to accommodate the mediastinal structures.

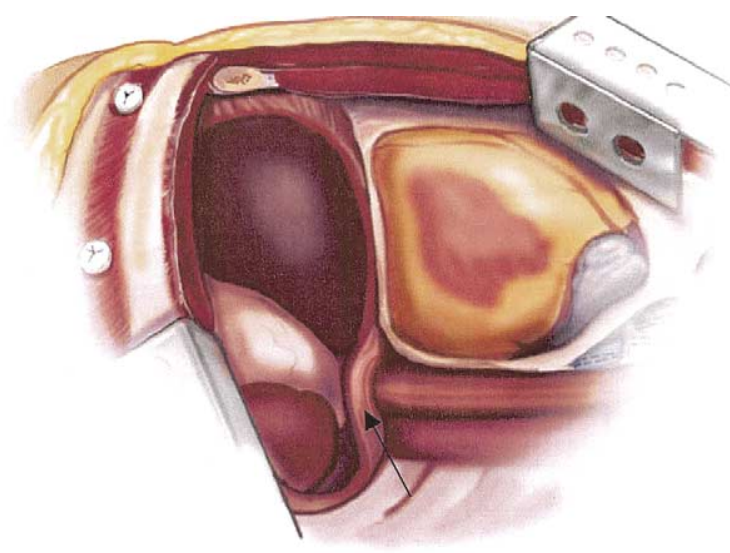

Figure 8. A rim of the diaphragmatic crus is left on the esophagus and stomach (arrow), which decreases the incidence of gastric herniation during left EPP.

diaphragmatic patch to the inferior cut edge of the pericardium. Care should also be taken to suture the patch to the cut edge of the pericardium instead of other weaker tissues to avoid cardiac herniation (Figure 3).

Although myocardial infarction is rare, pericarditis is frequently seen in association with diffuse ST-segment increases and high creatine phosphokinase, low MB fraction, and high troponin levels. These tend to resolve within 48 hours.

Cardiac arrest within 10 days postoperatively requires emergency thoracotomy (sometimes in the intensive care unit), open cardiac massage, and pericardial patch removal. Because the heart has shifted out of the midline, it cannot be compressed by the sternum and vertebral column, as normally occurs in closed chest massage. Additionally, emergency thoracotomy permits immediate drainage of un-

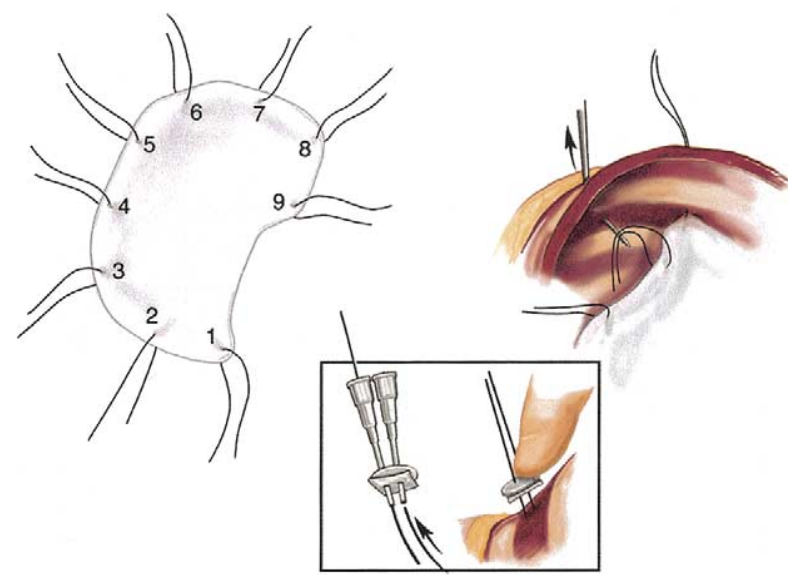

Figure 9. Buttons and a small piece of PTFE are used to secure the patch to the chest wall and prevent the sutures from pulling through the patch. Sutures are threaded with the use of intravenous angiocatheters and then tied, avoiding excess tension.

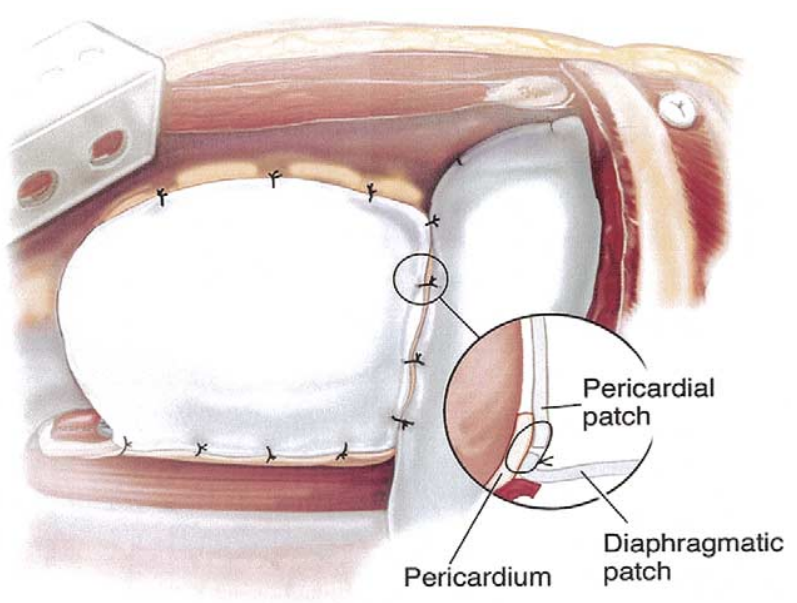

Figure 10. Both patches should be sutured to the cut edge of the pericardium medially and the chest wall anterolaterally and posteriorly. Failure to do so will result in cardiac or gastric herniation.

drained pericardial fluid and compressive fluid within the operative pleural space, removal of potentially constraining patches, and reduction of cardiac herniation, which occurs when the ventricles have herniated through the pericardial patch and pericardial defect, if present. Typically, the pericardium compresses the ventricular inflow at the atrioventricular groove, causing a sudden and catastrophic reduction in cardiac output. All members of the postoperative care team must be educated on this point to avoid losing valuable time performing futile closed chest compressions. After successful resuscitation, the patient is immediately returned to the operating room for pulsed irrigation cleanout of the 
chest cavity and surgical correction of the cause of the cardiac arrest.

\section{Pulmonary Complications}

Pulmonary complications include prolonged intubation (26/ 328 [7.9\%] patients), aspiration (9/328 [2.7\%] patients), acute respiratory distress syndrome (ARDS; 12/328 [3.6\%] patients), and the need for tracheostomy (6/328 [1.8\%] patients). These complications are closely associated with vocal cord paralysis, which occurred in $22(6.7 \%)$ of 328 patients.

A high index of suspicion is needed to diagnose and treat vocal cord paralysis. Patients who had extensive disease resected in high-risk areas for recurrent nerve injury (ie, aortopulmonary region and the subclavian vessels) are scoped postoperatively, even in the absence of symptoms such as a hoarse voice or poor cough. Vocal cord weakness places the patient at high risk for aspiration after pneumonectomy, which is frequently life-threatening. Therefore we recommend immediate injection of the vocal cords with gel foam when the condition is diagnosed. ${ }^{6}$

Aggressive diuresis postoperatively is essential to avoid pulmonary edema, and liberal use of bronchoscopy is needed to clear retained secretions.

Excessive mediastinal shift away from the pneumonectomy space into the contralateral hemithorax can result in decreased lung volume, retained secretions, and respiratory compromise. A red rubber catheter is left in the pneumonectomy space for 3 days, and fluid is evacuated as needed to ensure a midline mediastinum and contralateral lung expansion (Figure 4).

\section{Renal, Hematologic, and Infectious Complications}

Renal failure was seen in 9 (2.7\%) of 328 patients and is generally associated with ARDS, multiple organ failure, and death.

Deep vein thrombosis was recognized in 21 (6.4\%) of 328 patients, with 5 (1.5\%) of 328 patients having pulmonary embolism. Because a pulmonary embolus to a single remaining lung is always life-threatening, we have adopted an aggressive screening protocol, obtaining noninvasive vascular studies on all patients during the seventh postoperative day. We have a low threshold for using pulmonary embolus computed tomographic scans in patients with moderate symptoms or minor oxygen desaturations and have recently been diagnosing pulmonary emboli with a greater frequency.

Postoperative empyema has been unusual (8/328 [2.4\%] patients) in our experience. Infection in a chest with 2 prosthetic grafts can be catastrophic. Clinical signs of infection are often lacking and cultures may fail to detect anaerobic infection. Our prevention regimen includes pulsed irrigation of the pneumonectomy cavity with a high- pressure 9-L lavage intraoperatively. We use a small, red rubber catheter placed through the wound rather than standard-size chest tube placement through a separate incision to reduce potential contamination along the outside of the catheter. A triple intravenous antibiotic regimen, including cefazolin (Ancef; Smith Kline Beecham, Philadelphia, Pa), levofloxacin (Levaquin; Ortho-McNeil, Raritan, NJ), and metronidazole (Flagyl; Searle, Skokie, Ill), is used for 5 days perioperatively because prosthetic materials are used for the pericardial and diaphragmatic reconstruction.

When empyema does occur, management is dependent on the time of presentation. Patch removal and a traditional Clagett window will delay adjuvant chemoradiation therapy. Open chest management complicated by tumor recurrence is a dreaded complication. We have successfully used thoracoscopically guided closed chest management with full debridement of all fibrin and pulsed irrigation of the pleural cavity without patch removal. ${ }^{7}$ This is followed by postoperative irrigation for 5 days (Figure 5). The closed approach for postoperative empyemas was successful in 3 of the 4 patients who underwent this procedure. This technique is applied to patients who have empyema without bronchopleural fistulas during the first postoperative month. Patients who have empyema months to years after resection are usually managed with Clagett windows because they tend to present later in the course of their disease. If a Clagett window is deemed necessary because of the amount of infection, staged removal of the prosthetic polytetrafluoroethylene (PTFE) patches (Gore-Tex; W. L. Gore \& Associates, Inc, Flagstaff, Ariz) can prevent overshift of the mediastinum (Figure 6). Removal of the patches before mediastinal stabilization will result in hemodynamic compromise. The staged removal of the PTFE patch is used for patients who present early (ie, in the first 2 weeks, before the mediastinum has become scarred in) with empyema that is deemed unmanageable with thoracoscopic lavage or is complicated with bronchopleural fistulas. The Clagett window is created, leaving the patches in place. After 2 to 3 weeks of dressing changes, the mediastinum is sufficiently scarred to permit the patches to be removed. In our practice we tend to reserve this procedure for patients with bronchopleural fistulas. It is such a rare complication during the early postoperative period that we do not have an extensive experience or comparison between the various approaches to this complication.

The presence of a bronchopleural fistula necessitates drainage of the cavity with a Clagett window to protect the contralateral lung. This has only occurred twice in our experience $(0.6 \%)$.

\section{Technical Complications}

Our pericardial and diaphragmatic reconstructions have undergone constant evolution over the last 15 years. Our 
current method involves the use of the impermeable PTFE patch, $0.1 \mathrm{~mm}$ for the pericardium and $2 \mathrm{~mm}$ for the diaphragm. The impermeable diaphragm patch prevents excess fluid accumulation in the chest. Two patches are used and stapled with a TA stapler (proximate TX linear stapler, Ethicon, Inc, Somerville, NJ), creating a dynamic patch at the center with less tension at the sutures. The patch is then tailored to accommodate the mediastinal structures (Figure 7).

A rim of the diaphragmatic crus is left on the esophagus and stomach, decreasing the incidence of gastric herniation (Figure 8).

The patch should be sutured to the cut edge of the pericardium medially and the chest wall anterolaterally and posteriorly (Figure 9). The patch should be loose because a tight patch will predispose to herniation. Daily chest $\mathrm{x}$-ray films should be compared with the initial postoperative film. The mere suggestion of gastric or bowel herniation with complex gas patterns in the operative hemithorax should be rapidly assessed by means of computed tomographic scanning, and reoperation should occur as soon as it is recognized to avoid ischemic perforation and spillage of the bowel contents.

The method of securing the patch to the chest wall was developed in response to oncologic and technical issues. Bulky tumors necessitate resection of the entire diaphragm, leaving only a small rim of the diaphragm that is insufficient for securing the patch. We have no oncologic data to compare diaphragmatic resection techniques.

In addition, the diaphragmatic reconstruction technique was adjusted from the previous single-patch technique to the current double-patch technique as a consequence of concerns about gastric herniation. Patients with ileus seem to be particularly at risk because of increased intra-abdominal pressure and tension on the patch. The 2-patch method was used in an attempt to recreate the dome shape of the diaphragm. We have no laboratory data to support the superiority of the 2-patch technique. However, when we compared 2 groups of consecutive patients, the patch dehiscence incidence of $6(12 \%)$ of 50 with the old technique was reduced to 1 (3.8\%) of 26 with the 2-patch technique.

As stated previously, the pericardial patch is used for both right and left reconstructions to avoid the development of constrictive physiology. The pericardial patch should always be sutured to the pericardium because other tissues will not hold, and cardiac herniation might result in cardiac arrest (Figure 10).

Meticulous hemostasis is crucial because excessive bleeding can also result in mediastinal shift. Chylothorax remains rare, but an excessive rate of fluid accumulation in the pneumonectomy cavity can be an important clue. Treatment can now be facilitated with percutaneous embolization.

\section{Discussion}

The previous experience with EPP for mesothelioma reported by Butchart and colleagues ${ }^{1}$ was accompanied by an almost prohibitive mortality rate. The modern experience with EPP as a treatment for locally advanced cancer began in the 1980s, with reported mortality rates ranging from $6 \%{ }^{8}$ to $15 \% .^{3}$ Mortality rates have continued to decrease in the 1990 s, with some authors reporting up to a $50 \%$ reduction over previous mortality rates at their institutions. ${ }^{4,9}$ The Brigham strategy has been to continuously refine the surgical technique and to prevent and aggressively seek out complications at an early stage, when they are easier to treat and their physiologic effect on this tenuous group of patients remains minimal. This strategy has permitted the expansion of operative criteria to include patients who a few years ago would have been deemed inoperable.

The diaphragmatic and pericardial reconstructions are crucial to avoiding gastric and cardiac herniation. Our aggressive screening for vocal cord paralysis, thrombotic complications, and liberal use of bronchoscopy has helped a great deal in achieving low mortality rates because ARDS and cardiovascular complications, such as pulmonary embolus, remain the leading causes of death. The management of empyema and cardiac arrest is unique in these patients.

Most of these strategies remain empiric in nature. One could draw conclusions from randomized studies; however, the relatively small group of patients undergoing EPP every year compared with the number of patients undergoing lobectomies for lung cancer, for example, does not permit randomized studies to be conducted for testing these strategies.

This review represents our institution's 20-year experience from 1980 through 2000 and demonstrates the constant evolution of our operative technique, which has resulted in a steady reduction in postoperative mortality. The operative procedure discussed here demands intraoperative and postoperative vigilance from the care team for early signs of complications. The knowledge we have accumulated over the last 20 years re-enforces the view that a multidisciplinary approach remains crucial in the perioperative period.

\section{References}

1. Butchart EG, Ashcroft T, Barnsley WC, Holden MP. Pleuropneumonectomy in the management of diffuse malignant mesothelioma of the pleura. Experience with 29 patients. Thorax. 1976;31(1):15-24.

2. Allen KB, Faber LP, Warren WH. Malignant pleural mesothelioma. Extrapleural pneumonectomy and pleurectomy. Chest Surg Clin North Am. 1994;4(1):113-26.

3. Rusch VW, Piantadosi S, Holmes EC. The role of extrapleural pneumonectomy in malignant pleural mesothelioma. A Lung Cancer Study Group trial. J Thorac Cardiovasc Surg. 1991;102(1):1-9.

4. Sugarbaker DJ, Flores RM, Jaklitsch MT, Richards WG, Strauss GM, Corson JM, et al. Resection margins, extrapleural nodal status, and cell type determine postoperative long-term survival in trimodality therapy 
of malignant pleural mesothelioma: results in 183 patients. $J$ Thorac Cardiovasc Surg. 1999;117(1):54-65.

5. Byrne JG, Karavas AN, Colson YL, Bueno R, Richards WG, Sugarbaker DJ, et al. Cardiac decortication (epicardiectomy) for occult constrictive cardiac physiology after left extrapleural pneumonectomy. Chest. 2002;122(6):2256-9.

6. Bhattacharyya N, Batirel H, Swanson SJ. Improved outcomes with early vocal fold medialization for vocal fold paralysis after thoracic surgery. Auris Nasus Larynx. 2003;30(1):71-5.

7. Hollaus PH, Lax F, Wurnig PN, Janakiev D, Pridun NS. Videothoracoscopic debridement of the postpneumonectomy space in empyema. Eur J Cardiothorac Surg. 1999;16(3):283-6.

8. Sugarbaker DJ, Mentzer SJ, Strauss G. Extrapleural pneumonectomy in the treatment of malignant pleural mesothelioma. Ann Thorac Surg. 1992;54(5):941-6.

9. Rusch VW, Rosenzweig K, Venkatraman E, Leon L, Raben A, Harrison L, et al. A phase II trial of surgical resection and adjuvant high-dose hemithoracic radiation for malignant pleural mesothelioma. $J$ Thorac Cardiovasc Surg. 2001;122(4):788-95.

\section{Discussion}

Dr Daniel L. Miller (Atlanta, Ga). Dr Sugarbaker, I would like to congratulate you on an excellent presentation and also congratulate you and your colleagues at the Brigham for compiling the largest series of EPPs in the world. Your hard work and refinement of the technique of EPP has allowed this procedure to be put back into the armamentarium of thoracic surgeons so we can help to improve the long-term survival of patients with mesotheliomas.

I have several questions for you. Although more than $60 \%$ of your patients experience a minor or major complication after EPP, your overall operative mortality rate was less than $4 \%$. What do you believe has been the single most important factor that has reduced your operative mortality: selection, operative technique, postoperative care? Probably it is going to be the combination of the 3 , but what do you believe has been the most important?

Dr Sugarbaker. Thanks very much. I would say that reduction in operative time through the years probably has had a large effect on getting these patients in and out of the hospital. Selection has also been very, very important with the use of magnetic resonance imaging and echocardiography preoperatively-everybody gets an echocardiogram. And we have detected some previously undetected myocardial dysfunction that would have led to, I think, postoperative complications. But I would say the most important factor has been the reduction in operative time from an average of about $6 \frac{1}{2}$ hours to slightly less than 3 hours now from beginning to end.

Dr Miller. Your incidence of postoperative empyema and bronchopleural fistula was extremely low at $2.4 \%$ and $0.6 \%$, respectively. Could you comment again on your technique for bronchial reinforcement, which you did not bring up during the talk, and also again about your technique of drainage of the pleural cavity. I know that you have switched to a red rubber catheter. Why did you do that? Also, please discuss again the pulsed irrigation of the chest, which I think is new to a lot of us in the audience.

Dr Sugarbaker. I will start with the reinforcement of the stump. I think the most important thing is, particularly on the left, to have a short stump so that you do not have dependent secretions. We use a parathymic-pericardial fat pad based on a proximal pedicle to cover each stump and suture it in place with 3.0 Vicryl sutures. I think that is a very, very important component. I know there is some disagreement from some others who do this procedure, but we believe it is part of the overall plan.
The red rubber catheter is a large no. 9 catheter. It is brought out through the wound, through a little Christmas tree and a stopcock. That should initially balance the chest and have the ability to remove fluid. And I would say one of the things that has also gone down is that we do not reoperate for bleeding as much as we used to. We just keep aspirating fluid, and the patients do eventually stop bleeding if they have a bleeding problem, although that has also, I think, gone down quite a bit with reduction in operative time. Therefore the red rubber catheter, I think, is a very nice little instrument to have there. It is diagnostic, it is therapeutic, and it comes out easily at about 3 days. The pulsed irrigator is simply the Water Pik irrigator. You have probably seen orthopedists do this for hips. We use a combination of sterile water and Betadine solution, and, as mentioned in the article, do a 6-L lavage at the end of the procedure. It has helped, I think, to reduce not only our bacteriologic contamination but also cytologic contamination of the wound.

Dr Miller. The data analysis that you included in this study was through 2000. What effects has the addition of hyperthermic pleural lavage had on your complication rate, especially in regard to pleural space problems and deep venous thrombosis?

Dr Sugarbaker. That is an excellent insight. Our AF rate has gone to $80 \%$. When you lavage the heart with hyperthermic cisplatinum at a high dose, it is irritating, and most patients have gone into AF, which is pushing us to explore some other means of prophylaxis against that. The other major complication is a $24 \%$ deep vein thrombosis rate, which, as you mentioned, I think is probably from the hyperthermic effect on the coagulation system with the blood heating up. The core temperatures do get up there to around $41^{\circ} \mathrm{C}$.

Dr Miller. Because of your high morbidity rate and so forth, you have always talked about trimodality therapy being very important. Because of this high morbidity rate, what percentage of your patients completes all 3 modalities?

Dr Sugarbaker. That is a good question. The data are that about $80 \%$ of patients received adjuvant therapy. Now, much of it is done back home. Two cycles, 1 cycle, 3 cycles; it is sometimes hard to get your hands around that data, so that is why I have not presented it.

Dr W. Roy Smythe (Houston, Tex). Dr Sugarbaker, we enjoyed your article and your teaching, and there is no doubt that those of us who have been doing this for the last 5 or 10 years are standing squarely on your shoulders, and we really appreciate all of your hard work.

Dr Sugarbaker. Thank you.

Dr Smythe. I have 2 brief questions. One, have you stratified these morbidities by stage, either your staging system or the new AJCC? Second, in addition to using electrocautery and the argon beam, have you considered using any pharmacologic intervention? Of course, with your recent experience with the deep vein thromboses, this might sound a little scary, but we have been using aprotinin for about the last 40 or 50 patients and have seen a significant decrease in blood product use, which we think translates to a lower pulmonary complication profile.

Dr Sugarbaker. I thank you very much for your kind comments. Let me start with your last question. We have looked at several pharmacologic ways in which we might improve and 
reduce blood loss, but we do have this problem now, particularly with the hyperthermic patients, of deep vein thromboses and a pulmonary embolism rate that is apparently going up. Therefore I think we are going to approach that with some trepidation.

Your other question I do not have an answer for, and that is, is there a staged distribution. The majority of patients are stage I or stage II by the Brigham staging system with N1 disease or N0 disease. The majority do not have N2 disease. Extrathoracic node positivity runs about $25 \%$. But I do not have a stratification of this morbidity, which is primarily reversible. I mean, most of it was $\mathrm{AF}$, which is reversible, but there is no question that it is significant.

Dr Raja M. Flores (New York, NY). I have a comment on pericardial reconstruction and a question. At our institution, we use Dexon mesh for several reasons for the pericardial reconstruction. One is that there is no need for fenestration; it is already porous. The other is that it has more give than PTFE, so when the heart becomes edematous, especially after intraoperative chemotherapy, there is more give and less chance of cardiac constriction. Third and most importantly is that it is absorbable, and therefore in the event of empyema, there is no need to pull the PTFE off of an already friable myocardium.

My question is with regard to AF prophylaxis. Have you started yet, and have you come into any problems? These patients are already hemodynamically unstable, they have an epidural catheter in, there is already potential for bleeding, and then when you add on to that calcium channel blockers or $\beta$-blockers, I would anticipate problems with hemodynamics.

Dr Sugarbaker. Thank you very much for your comments, Dr Flores. In terms of the pericardial reconstruction, that is an interesting concept to use an absorbable patch. The low incidence of empyema makes it sort of less of an issue in terms of removing the PTFE, which, as you know, cannot be wetted and usually comes off the heart without too much difficulty. 